\title{
Synchrony Generation in Recurrent Networks with Frequency-Dependent Synapses
}

\author{
Misha Tsodyks, Asher Uziel, and Henry Markram \\ Department of Neurobiology, Weizmann Institute of Science, Rehovot 76100, Israel
}

Throughout the neocortex, groups of neurons have been found to fire synchronously on the time scale of several milliseconds. This near coincident firing of neurons could coordinate the multifaceted information of different features of a stimulus. The mechanisms of generating such synchrony are not clear. We simulated the activity of a population of excitatory and inhibitory neurons randomly interconnected into a recurrent network via synapses that display temporal dynamics in their transmission; surprisingly, we found a behavior of the network where action potential activity spontaneously self-organized to produce highly synchronous bursts involving virtually the entire network. These population bursts were also triggered by stimuli to the network in an all-or-none manner. We found that the particular intensities of the external stimulus to specific neurons were crucial to evoke population bursts. This topographic sensitivity therefore depends on the spectrum of basal discharge rates across the population and not on the anatomical individuality of the neurons, because this was random. These results suggest that networks in which neurons are even randomly interconnected via frequency-dependent synapses could exhibit a novel form of reflex response that is sensitive to the nature of the stimulus as well as the background spontaneous activity.

Key words: synaptic plasticity; action potential encoding; neural network; modeling; cortical column; spike timing
Although increased firing rate of a single neuron is the clearest signature of its participation in a particular processing, growing evidence indicates that temporal coherence in the activity of groups of neurons may be an important component of the neuronal code. Indeed, throughout the neocortex, the spiking activity of groups of cells has been found to exhibit various patterns of synchrony, during both spontaneous activity and under sensory stimulation (Murphy et al., 1985; Gray et al., 1989; Vaadia and Aersten 1992; Sillito et al., 1994; Riehle et al., 1997; Steriade and Contreras, 1998). Synchrony could allow the information about external stimuli to be coded in the temporal relation between the spiking of different neurons (Abeles, 1991; Hopfield, 1995) or provide the basis for binding different features belonging to the same object (Singer and Gray, 1995).

The mechanisms of inducing synchronous firing in groups of cells are not yet directly accessible to experimental study. A pair of neurons could either synchronize via direct synaptic connection between them or as a result of a common input. Anatomical evidence indicates that most of the synaptic contacts a cortical cell receives are in fact originating from the cells that are located in the same cortical area (Ahmed et al., 1994). It is therefore reasonable to assume that much of the observed synchronization is generated locally as a consequence of the population dynamics.

Synchrony generation by networks of interconnected neurons is a subject of many theoretical and numerical studies. For example, it is easy to construct a network in which firing of individual neurons is perfectly locked to each other (Mirollo and Strogatz, 1990). On the other extreme, the network could be in the regimen

Received July 8, 1999; revised Oct. 19, 1999; accepted Oct. 21, 1999.

The study was supported by grants from the Israeli Academy of Science, the Office of Naval Research, Binational Science Foundations, and the Human Frontier Science Program Organization.

Correspondence should be addressed to Misha Tsodyks at the above address. E-mail: bnmisha@wicc.weizmann.ac.il.

Copyright (C) 1999 Society for Neuroscience 0270-6474/99/200001-05\$15.00/0 of asynchronous activity with uncorrelated firing of individual neurons (Abbott and van Vreeswijk, 1993). In this state, the precise timing of individual spikes is not important, and the activity of the neuron can be adequately described by its firing rate. An intermediate regimen of synchronized chaotic activity was obtained in the network of Hansel and Sompolinsky (1996), where only a fraction, albeit a large one, of spikes in pairs of neurons was synchronized on the time scale of few milliseconds.

Sharp synchronization between the spiking activity of pairs of neurons was also reported in several experimental studies (Murphy et al., 1985; Vaadia and Aersten, 1992; Riehle et al., 1997). In these studies, however, the spikes were tightly locked for short epochs lasting some milliseconds, and the rest of the spikes were at most loosely correlated. This suggests that cortical networks can operate in a regimen in which asynchronous activity is intermittent with sharp synchronization on the time scale of single spikes.

Previous modeling studies have not considered potential effects of nonlinear (frequency-dependent) synapses on synchronization of neuronal activity in recurrent networks. In the neocortex, diversity of depressing and facilitating synapses has been reported (Thomson and Deuchars, 1994; Markram et al., 1998). Nonlinear

This article is published in The Journal of Neuroscience, Rapid Communications Section, which publishes brief, peerreviewed papers online, not in print. Rapid Communications are posted online approximately one month earlier than they would appear if printed. They are listed in the Table of Contents of the next open issue of JNeurosci. Cite this article as: JNeurosci, 2000, 20:RC50 (1-5). The publication date is the date of posting online at www.jneurosci.org.

http://www.jneurosci.org/cgi/content/full/3810 
synaptic transmission was implicated in shaping the signaling between neocortical neurons (Grossberg, 1969; Thomson and Deuchars, 1994; Abbott et al., 1997; Tsodyks and Markram, 1997; Markram et al., 1998). In particular, synaptic depression, which is ubiquitous in connections between pyramidal neurons, enables transmission of signals reflecting the synchrony in presynaptic ensembles (Senn et al., 1998). In the current study, we demonstrate that when nonlinear synaptic transmission is incorporated into recurrent networks, intermittent activity with short-time synchrony naturally emerges. This comes about as a result of occasional upswings in the network population activity, during which most of the neurons fire an action potential within a period of several milliseconds. Moreover, during these periods, the neurons tend to fire in a specific temporal order, which depends on the distribution of the average firing rates of the neurons.

\section{MODEL}

We simulated a recurrent network of 400 excitatory and 100 inhibitory neurons. The neurons were connected at random with a probability of a contact between a pair of neurons taken to be $10 \%$ in accordance with anatomical data (Abeles, 1991). Neurons were modeled as leaky integrate-and-fire units widely used in these kinds of simulations (Tuckwell, 1988). Each unit was described by its voltage membrane potential, which evolved according to the circuit equation:

$$
\tau \frac{d V}{d t}=-V+R_{\mathrm{in}}\left(I_{\mathrm{syn}}+I_{\mathrm{b}}\right)
$$

where $\tau$ denotes the membrane time constant of a neuron, $I_{\text {syn }}$ represents the synaptic current mediated by internal connections, and $I_{\mathrm{b}}$ stands for the nonspecific background current provided by the distant brain areas. In the following, we incorporated the input resistance of the neuron, $R_{\mathrm{in}}$, into the currents, which were therefore measured in units of voltage (millivolts). The membrane potential $V$ was calculated relative to a resting level for a given neuron. Each time the membrane potential of a neuron reached threshold, a spike was emitted, and the voltage was put to a reset value after an absolute refractory period. In our simulations $\tau=30 \mathrm{msec}$, and the membrane threshold and reset values were 15 and $13.5 \mathrm{mV}$, respectively. Excitatory neurons had a refractory period of 3 $\mathrm{msec}$, and inhibitory neurons had a refractory period of $2 \mathrm{msec}$. The background current, $I_{\mathrm{b}}$, had a constant value for each neuron, randomly distributed across the network. We chose a uniform distribution centered at the threshold level with a range of $0.05 \mathrm{mV}$; this resulted in the basal firing rates of the excitatory neurons being between 1 and $20 \mathrm{~Hz}$, with an average of $7 \mathrm{~Hz}$. The synaptic current, $I_{\text {syn }}$, was modeled as a sum of postsynaptic currents (PSCs) from all of the other neurons in the network which have connections targeting the given neuron (i):

$$
I_{\mathrm{syn}}(\mathrm{i})=\sum_{\mathrm{j}} A_{\mathrm{ij}} y_{\mathrm{ij}}(t) .
$$

We used the phenomenological description of nonlinear synapses developed by Markram et al. (1998) and Tsodyks et al. (1998), which was shown to capture well the experimentally observed properties of neocortical connections. In this model, $A_{\mathrm{ij}}$ is a parameter describing the absolute strength of the synaptic connection between neurons $j$ (presynaptic neuron) and i (postsynaptic neuron). The effective synaptic strength is determined by the factor $y_{\mathrm{ij}}$, which describes the contribution to a synaptic current of neuron i because of PSCs from a neuron $\mathrm{j}$. It evolves according to the system of kinetic equations:

$$
\begin{aligned}
& \frac{d x}{d t}=\frac{z}{\tau_{\mathrm{rec}}}-u x \delta\left(t-t_{\mathrm{sp}}\right) \\
& \frac{d y}{d t}=-\frac{y}{\tau_{\mathrm{I}}}+u x \delta\left(t-t_{\mathrm{sp}}\right) \\
& \frac{d z}{d t}=\frac{y}{\tau_{\mathrm{I}}}-\frac{z}{\tau_{\text {rec }}},
\end{aligned}
$$

where $x, y$, and $z$ are the fractions of synaptic resources in the recovered, active, and inactive states, respectively, $t_{\mathrm{sp}}$ denotes the timing of presyn- aptic spikes, $\tau_{\mathrm{I}}$ is the decay constant of PSCs, and $\tau_{\text {rec }}$ is the recovery time from synaptic depression. These equations describe the use of synaptic resources by each presynaptic spike (a fraction $u$ of the available resources $x$ is used by each presynaptic spike). A running variable, $u_{\mathrm{ij}}$, describes the effective use of the synaptic resources of the synapses, which is analogous to the probability of release in the quantal model (Markram et al., 1998). In facilitating synapses, it is increased with each presynaptic spike and returns to the baseline value with a time constant of $\tau_{\text {facil }}$ :

$$
\frac{d u}{d t}=-\frac{u}{\tau_{\text {facil }}}+U(1-u) \delta\left(t-t_{\mathrm{sp}}\right),
$$

where the parameter $U$ determines the increase in the value of $u$ with each spike. If $\tau_{\text {facil }} \rightarrow 0$, facilitation is not exhibited, and $u$ is identical to $U$ for each spike, as is the case with depressing synapses between excitatory pyramidal neurons (Tsodyks and Markram, 1997). The values of the synaptic parameters were assigned as follows. First, average values were specified for the parameters for each type of the connections; then for each connection the actual value was chosen from a Gaussian distribution with a corresponding mean and with a SD that equals half that mean. The average values of the parameters used in the simulations were $A(\mathrm{ee})=1.8 \mathrm{mV} ; A(\mathrm{ei})=5.4 \mathrm{mV} ; A(\mathrm{ie})=7.2 \mathrm{mV} ; A(\mathrm{ii})=7.2 \mathrm{mV}$; $U(\mathrm{ee})=U(\mathrm{ei})=0.5 ; U(\mathrm{ie})=U(\mathrm{ii})=0.04 ; \tau_{\text {rec }}(\mathrm{ee})=\tau_{\text {rec }}(\mathrm{ei})=800 \mathrm{msec}$; $\tau_{\text {rec }}($ ie $)=\tau_{\text {rec }}($ ii $)=100 \mathrm{msec} ; \tau_{\text {facil }}($ ie $)=\tau_{\text {facil }}($ ii $)=1000 \mathrm{msec}$; and $\tau_{\text {I }}=$ $3 \mathrm{msec}$. In control simulations we verified that the qualitative behavior of the network is robust against independently changing the average values by $50 \%$.

\section{RESULTS \\ Network dynamics}

An epoch of network simulations is illustrated in Figure 1. The raster plot of neuronal activity (Fig. $1 A$ ) and the average network activity (Fig. $1 B$ ) indicate that the network dynamics exhibit short intervals of highly synchronous activity in which large groups of neurons fire successively-population bursts (PB). With the choice of the parameters values given in Model, the duration of the PB was $<15 \mathrm{msec}$. On average, in each burst $95 \%$ excitatory and $98 \%$ inhibitory neurons participated, and the spikes were distributed such that $63 \%$ of them were emitted within $5 \mathrm{msec}$ around the peak of the burst, and $15 \%$ were emitted within $1 \mathrm{msec}$ (Fig. $1 B$ ). During the PBs, $95 \%$ of the neurons fired once, and the rest of the neurons emitted one or two additional spikes at the end of the burst. The rate of the bursts was $0.97 \pm 0.4 \mathrm{~Hz}$.

\section{Mechanisms of population burst}

Once a population burst occurs, it is easy to understand why it terminates. Because the synapses between excitatory neurons are depressing, the average effective strength of these connections quickly declines during the PB (Fig. $1 C$ ), and the neuron discharge times become independent of each other. After the burst the strength of the connections slowly recovers, because the subsequent asynchronous activity is characterized by low firing rates. This recovery of strength augments the probability that the firing of an excitatory cell will drive other cells to fire, and eventually, after the network has reached a certain threshold state, an excitatory cell can recruit other cells to generate a fast-developing synchronous activity.

To confirm the crucial role of synaptic depression between excitatory neurons in governing the population bursts, we eliminated nonlinearity from all other connections. Indeed, we observed the same qualitative behavior of the network activity (data not shown). In fact, PBs could even be observed in a network without inhibitory interneurons, provided the strength of recurrent excitation is adjusted appropriately (data not shown). We found, however, that inhibition allowed the PBs to occur for a wider range of parameters. Because synaptic depression is re- 

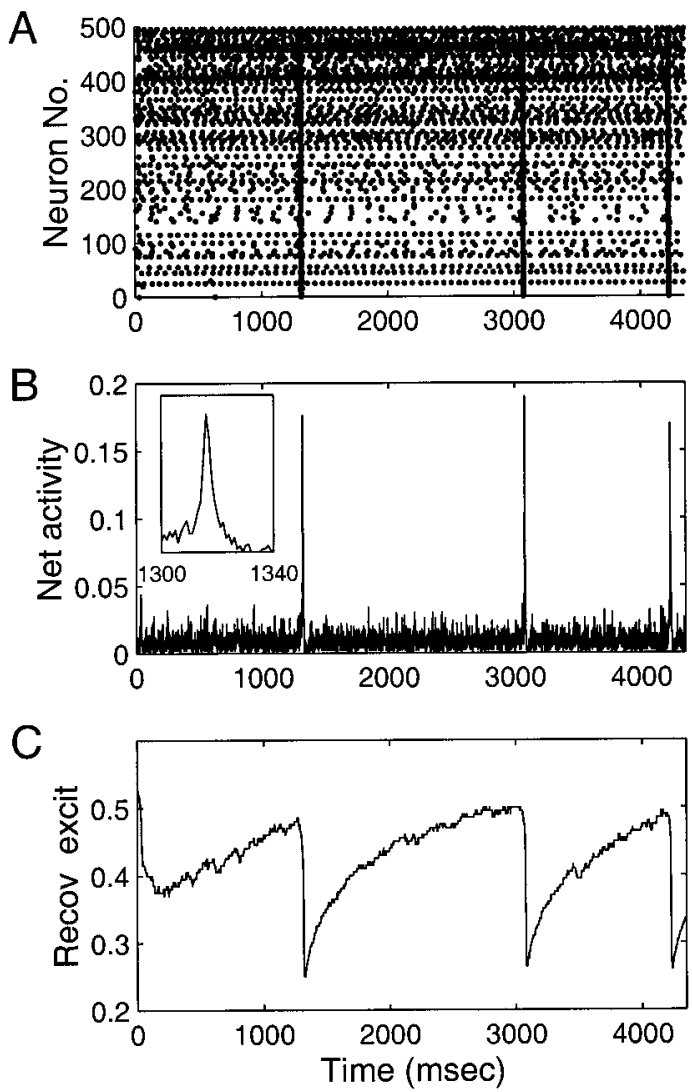

Figure 1. Network dynamics. $A$, Spike trains of every fifth neuron in a time window of $4.3 \mathrm{sec}$. For each neuron, a dot is put at each time the neuron emitted an action potential. $B$, Network activity, computed as the relative number of neurons that fire an action potential during consecutive bins of $1 \mathrm{msec}$. Inset, Network activity during the time window of 40 msec around the population burst. $C$, Fraction of synaptic resources in the recovered state, describing the effective strength of depressing synapses (see Model), averaged over all connections between excitatory neurons.

sponsible for PBs, it is reasonable to assume that the neurons with the lower firing rates are important in their generation. This is because the effective strength of a depressing synapse is decreasing with the firing rate of the presynaptic neuron. To clarify this issue, we ordered the excitatory neurons according to their average basal firing rates (Fig. $2 A$ ) over the interval of $20 \mathrm{sec}$. We then selectively eliminated groups of 30 excitatory neurons, ranked according to their firing rates, and counted the number of remaining bursts in an interval of $20 \mathrm{sec}$ after the start of the simulations. As shown in Figure $2 B$, the bursts disappear completely if the group of neurons with the firing rates from 1.3 to 2.5 $\mathrm{Hz}$ is eliminated. We explain this result by the fact that these neurons not only have low firing rates, and therefore effectively strong excitatory synapses, but also are close enough to threshold to trigger the avalanche of the firing activity leading to the crescendo $\mathrm{PB}$.

\section{Population response to external stimulation}

Spontaneous PBs resulted only when the connections between excitatory neurons were strong enough. External stimuli, however, could evoke population bursts even when connections were weak. To demonstrate this possibility, we uniformly reduced the absolute strength of all the synapses in the network until the PBs disappeared. We then studied the response of the network to
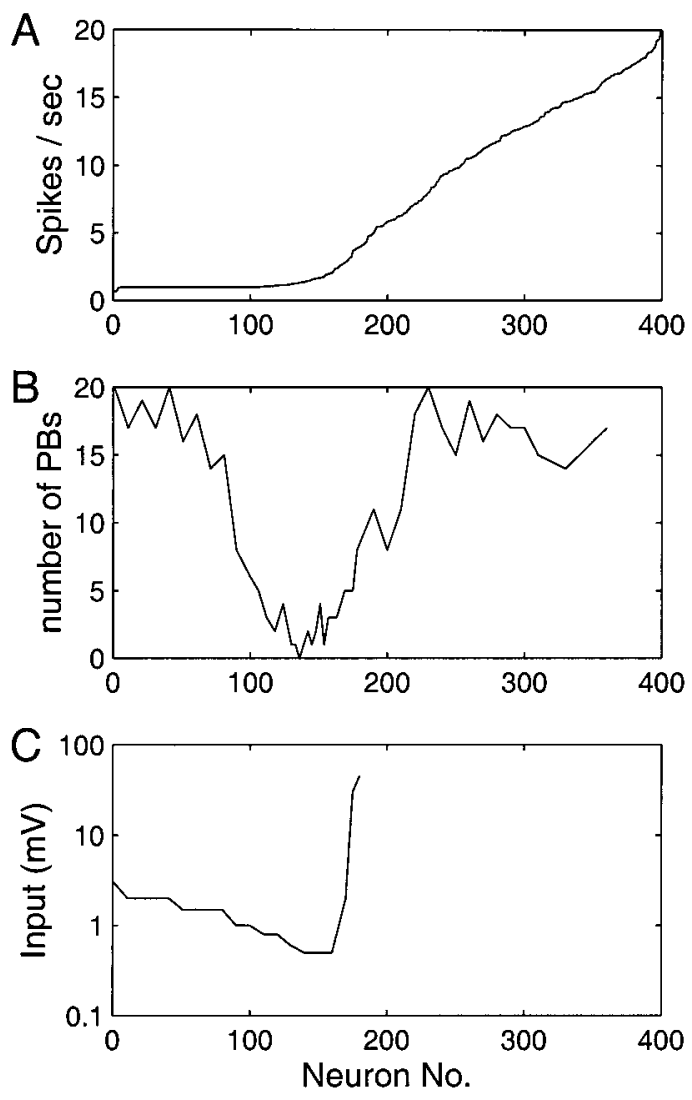

Figure 2. Mechanism of population bursts. A, Average firing rates of the excitatory neurons in the network, in an ascending order, computed over the interval of $20 \mathrm{sec}$. $B$, Number of population bursts over the same interval, which are observed in simulations in which a group of 30 neurons is taken out of the network, starting from the neuron indicated on the horizontal axis. $C$, Network with the absolute strength $(A)$ of all connections reduced by one-third of their original values, resulting in the disappearance of spontaneous population bursts. For each group of neurons, an input pulse lasting $5 \mathrm{msec}$ is applied with a frequency of $1 \mathrm{~Hz}$. The minimal amplitude of the input (in millivolts) that is required to reliably evoke a population burst is plotted for each group of 30 targeted neurons.

sharp (5 msec) input pulses of various amplitudes with a frequency of $1 \mathrm{~Hz}$, targeting groups of 30 neurons. In Figure $2 C$ the minimal amplitude of the pulse needed to evoke a PB in the network is plotted against different choices of the targeted neurons. As the firing rate of targeted neurons increased, the intensity of the pulse required for PB decreased gradually, reaching the minimum for about the same group of neurons, which are mostly responsible for spontaneous burst generation (Fig. 2, compare $B$, $C$ ). For groups of targeted neurons with higher firing rates, the input intensity required to generate the bursts increased sharply. Eventually, no bursts could be evoked at any input intensity when the targeted neurons had rates of $>5 \mathrm{~Hz}$. The network is therefore sensitive to the precise topography of the input stimulus; i.e., the ability of inputs with the identical amplitude to evoke a network response depends on the basal firing rates of the targeted neurons. To illustrate this sensitivity, we show the response of the network to inputs with the amplitude of $0.5 \mathrm{mV}$. When applied to a group of neurons with low basal rates, the input evoked a full-fledged $\mathrm{PB}$, whereas only minor response was observed when neurons with higher basal rates were targeted (Fig. 3A,B). 
A

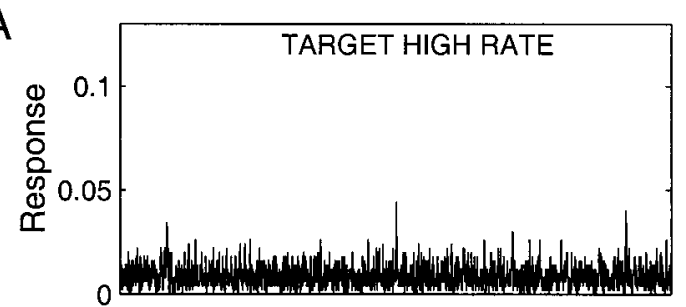

B

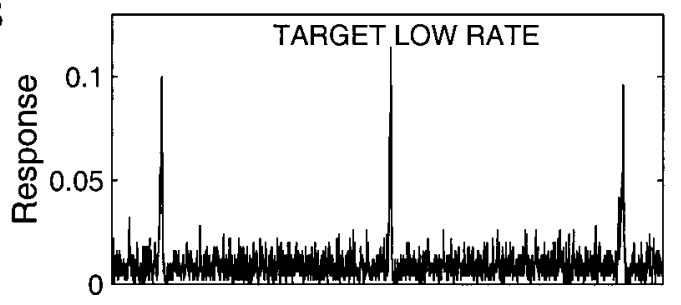

C

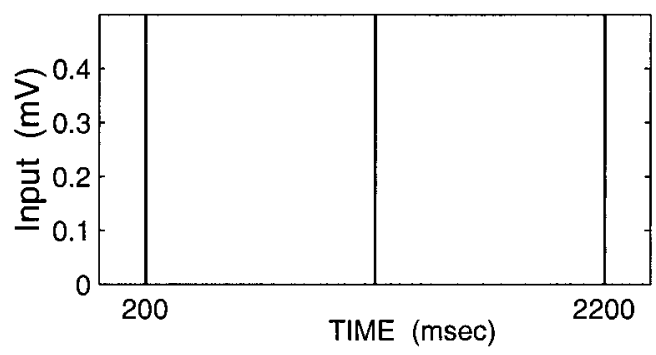

Figure 3. Sensitivity of the network to the precise topography of the stimulus. Network activity in response to input pulses of an amplitude of $0.5 \mathrm{mV}$ and a duration of $5 \mathrm{msec}$ is shown, targeting 30 neurons with rates of $3.5-5.9 \mathrm{~Hz}(A)$ and $1.1-2.9 \mathrm{~Hz}(B)$.

\section{Temporal relationship between neuronal firing}

The simulation results indicate that the network exhibits qualitatively different activity patterns during and between the PBs. To illustrate this difference, we computed the cross-correlation function (CCF) between the firing of different pairs of neurons, over the period of $1000 \mathrm{sec}$. In Figure $4 A$ we show an example of CCF for two neurons having the firing rates close to $10 \mathrm{~Hz}$. A sharp peak at zero time difference for the CCF is a direct result of the PBs during which both of the neurons fire within a short time interval. If one excludes the spikes emitted during the PBs, the resulting CCF does not exhibit any substantial peaks, indicating that between the bursts the firing of neurons remains essentially asynchronous. The area under the central peak in the CCF equals $10 \%$ of the overall number of spikes emitted by each of the neurons, which corresponds to the relative number of spikes emitted during the PBs. In Figure $4 B$, we show another pair, this time with one of the neurons having a low firing rate of $\sim 2 \mathrm{~Hz}$. A small negative bias of $4 \mathrm{msec}$ in the CCF can be detected, indicating that the neuron with the lower firing rate systematically fired before the neuron with the higher firing rate. This is consistent with the previous observation that the neurons with low firing rates play a crucial role in generating the PBs. Finally, Figure $4 C$ illustrates a pair of neurons with low rates, which exhibit a CCF with two sharp peaks at both positive and negative time difference. The shape of CCFs indicates that there exists a statistically reproducible timing relation between the firing of different neurons during a PB. To illustrate this relation, we computed, for each neuron in the network, the CCF between the
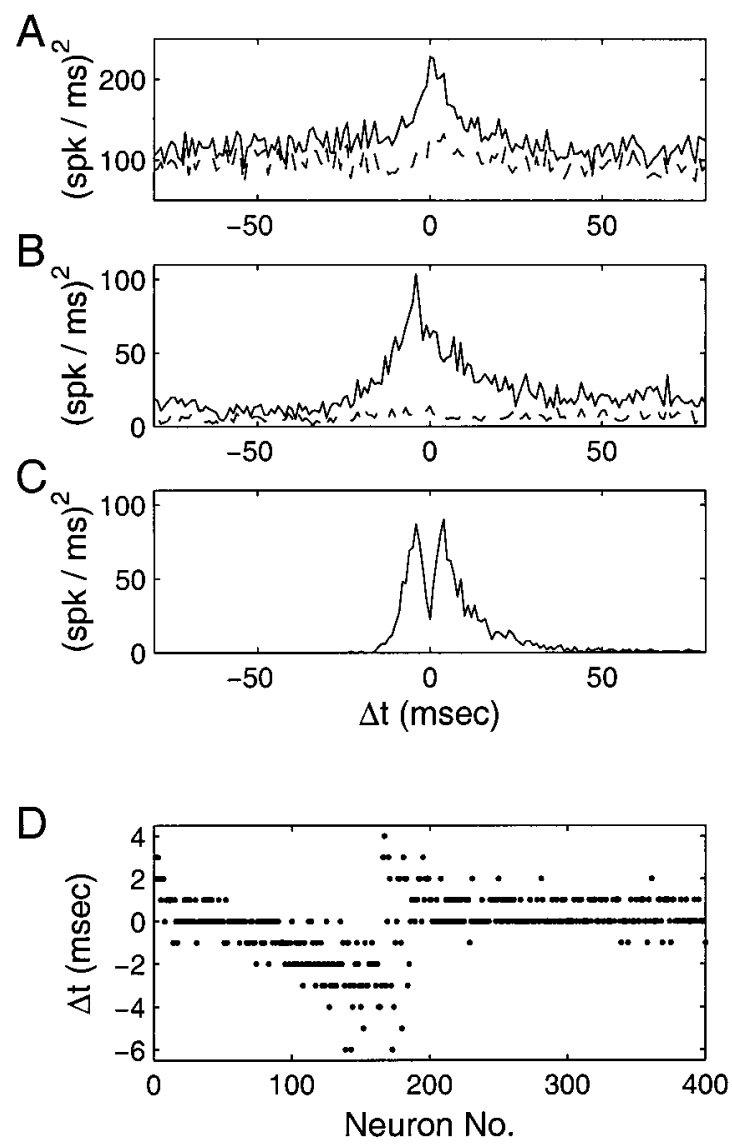

Figure 4. Temporal relationship between neuronal firing. A, Crosscorrelation function between the activities of two neurons with similar rates in the middle of the rate distribution (10 and $12 \mathrm{~Hz}$ ). Solid line, Cross-correlation function computed over the period of $1000 \mathrm{sec}$ of activity; dashed line cross-correlation function computed over the same period but subtracting the spikes emitted during the population bursts of the network. $B$, Same for a pair of neurons with rates of 1.7 and $12 \mathrm{~Hz}$. $C$, Pair of neurons with low rates of 2.6 and $1 \mathrm{~Hz}$. D, For each neuron in the network, the cross-correlation function between its spike train and the times of the maximums of population bursts was computed, and the time difference at which the cross-correlation function is at its maximum is plotted.

spike train of the neuron and the peaks of the population bursts and marked the time at which CCF was at its maximum (Fig. 4D). As seen by comparison between Figures $2 B$ and 4D, the neurons that are responsible for $\mathrm{PB}$ generation systematically fire in the advanced phase of the PB.

\section{Conditions for the occurrence of population bursts}

In the current study, we concentrated on the novel form of network activity, characterized by (spontaneous or evoked) sharp population bursts. As follows from the analysis, the necessary condition for this regimen is a broad distribution of the neuronal basal firing rates, which must include a substantial fraction of low-rate neurons. Indeed, if the rate distribution is shifted toward higher rates, e.g., because of increased tonic input, the bursts eventually disappear. Another condition concerns the strength of recurrent excitatory connections. We found that increasing the strength of the connections beyond a certain range $[\mathrm{A}(\mathrm{ee})>2.45$ $\mathrm{mV}$ for our network] led to broadening of the bursts such that single neurons were firing multiple spikes within each $\mathrm{PB}$. We believe that this happens because several spikes are now needed 
before synaptic depression weakens the recurrent excitation sufficiently. In this regimen, bursts are also more regularly spaced in time, which might therefore correspond to slow cortical oscillations observed during natural sleep in cats and humans (Steriade, 1997). We emphasize, however, that also in this regimen, the neurons that trigger the PBs have very little activity between the bursts. Finally, because of computational constraints, most of the simulations were performed with small networks consisting of 500 neurons. We checked that PBs also occurred in larger networks with the same pattern of connectivity. The frequency and temporal regularity of PBs are complex functions of the network size and synaptic parameters. In particular, networks of increasing size with fixed synaptic strength tend to exhibit more rhythmic activity.

\section{DISCUSSION}

The simulations presented here demonstrate that networks of neurons interconnected with nonlinear synapses have a striking tendency to generate a special regimen of activity with population bursts intermittent with long periods of asynchronous activity. As a result of the PBs, the neurons exhibit synchronous firing characterized by a large fraction of neurons firing action potentials within a time window of a few milliseconds. Within this short window, the neurons fire with a particular temporal relationship, determined by their basal firing rates during the preceding activity. The network can also produce a PB in response to an external excitatory input. In this case, the strength of excitation, required to evoke the response, strongly depends on the basal firing rates of the targeted neurons. Thus, the network is characterized by a high sensitivity to the topography of the input stimulus.

We emphasize that synaptic depression between excitatory neurons plays a crucial role in generating this network behavior. We also emphasize that synchronization between neurons on the time scale of few milliseconds can be achieved in randomly connected networks, without any specific structures such as synfire chains (Abeles, 1991). The network simulated in this study has a random pattern of intrinsic synaptic connections. For example, it could represent a cortical minicolumn consisting of neurons with similar receptive field properties. We expect that in larger networks, such as a cortical hypercolumn, where connections between neurons reflect their receptive field properties, other regimens of activity, with PBs propagating between different minicolumns in a quasirandom manner, could exist. These possibilities are subjects of a further study. The conclusion of the analysis could be tested experimentally by recordings from multiple neurons in the same cortical area. The results of the simulations predict that groups of cells can generate tightly synchronous spikes on the scale of few milliseconds, intermittent with the long periods with no synchronization on this scale. This effect could be observed during either spontaneous firing or during the response to sensory stimulation. There should also exist a systematic temporal relation between the timing of spikes in pairs of neurons in relation to their average firing rate. In particular, the spikes of neurons with low firing rates should precede the spikes of neurons with higher rates.

We would like to speculate that PBs that seem to be a natural tendency in recurrent networks with nonlinear synapses could have a functional significance for cortical networks. Because long-term regulation of synaptic transmission was shown to be sensitive to relative timing of spikes between presynaptic and postsynaptic neurons (Markram et al., 1997), spontaneous PBs could influence the shaping of local functional connectivity in the cortex. The PBs evoked by the external input could provide the cortex with the ability for a fast and reliable processing of the sensory stimuli, in accordance with recent psychophysical and electrophysiological observations (Bair and Koch, 1996; Thorpe et al., 1996; Eggermont, 1999).

\section{REFERENCES}

Abbott LF, van Vreeswijk C (1993) Asynchronous states in networks of pulse-coupled oscillators. Phys Rev E 48:1483-1490.

Abbott LF, Varela JA, Sen K, Nelson SB (1997) Synaptic depression and cortical gain control. Science 275:220-224.

Abeles M (1991) Corticonics. New York: Cambridge UP.

Ahmed B, Anderson J, Douglas R, Martin K, Nelson J (1994) Polyneural innervation of spiny stellate neurons in cat visual cortex. J Comp Neurol 341:39-49.

Bair W, Koch C (1996) Temporal precision of spike trains in extrastriate cortex of the behaving macaque monkey. Neural Comput 8:1185-202.

Eggermont JJ (1999) The magnitude and phase of temporal modulation transfer functions in cat auditory cortex. J Neurosci 19:2780-2788.

Gray CM, Konig P, Engel AK, Singer W (1989) Oscillatory responses in visual cortex exhibit intercolumnar synchronization which reflects global stimulus properties. Nature 338:334-337.

Grossberg S (1969) On the production and release of chemical transmitters and related topics in cellular control. J Theor Biol 22:325-364.

Hansel D, Sompolinsky H (1996) Chaos and synchrony in a model of a hypercolumn in visual cortex. J Comp Neurosci 3:7-34.

Hopfield JJ (1995) Pattern recognition computation using action potential timing for stimulus representation. Nature 376:33-36.

Markram H, Lubke J, Frotscher M, Sakmann B (1997) Regulation of synaptic efficacy by coincidence of postsynaptic APs and EPSPs. Science 275:213-215.

Markram H, Wang Y, Tsodyks M (1998) Differential signaling via the same axon from neocortical layer 5 pyramidal neurons. Proc Natl Acad Sci USA 95:5323-5328.

Mirollo RE, Strogatz SH (1990) Synchronization of pulse-coupled biological oscillators. SIAM J Appl Math 6:1645-1657.

Murphy JT, Kwan HC, Wong YC (1985) Cross-correlation studies in primate motor cortex: synaptic interaction and shared input. Can J Neurol Sci 12:11-23.

Riehle A, Grun S, Diesman M, Aertsen A (1997) Spike synchronization and rate modulation differentially involved in motor cortical function. Science 278:1950-1953.

Senn W, Segev I, Tsodyks M (1998) Reading neuronal synchrony with depressing synapses. Neural Comput 10:815-819.

Sillito AM, Jones HE, Gerstein GL, West DC (1994) Feature-linked synchronization of thalamic relay cell firing induced by feedback from the visual cortex. Nature 369:479-482.

Singer W, Gray CM (1995) Visual feature integration and the temporal correlation hypothesis. Annu Rev Neurosci 18:555-586.

Steriade M (1997) Synchronized activities of coupled oscillators in the cerebral cortex and thalamus at different levels of vigilance. Cereb Cortex 7:583-604.

Steriade M, Contreras D (1998) Spike-wave complexes and fast components of cortically generated seizures. i. Role of neocortex and thalamus. J Neurophysiol 80:1439-1455.

Thomson AM, Deuchars J (1994) Temporal and spatial properties of local circuits in neocortex. Trends Neurosci 17:119-126.

Thorpe S, Fize D, Marlot C (1996) Speed of processing in the human visual system. Nature 381:520-522.

Tsodyks M, Markram H (1997) The neural code between neocortical pyramidal neurons depends on neurotransmitter release probability. Proc Natl Acad Sci USA 94:719-723.

Tsodyks M, Pawelzik K, Markram H (1998) Neural networks with dynamic synapses. Neural Comput 10:821-835.

Tuckwell HC (1988) Introduction to theoretical neurobiology. New York: Cambridge UP.

Vaadia E, Aersten A (1992) Coding and computation in the cortex: single-neuron activity and cooperative phenomena. In: Information processing in the cortex (Aertsen A, Braitenberg V, eds), pp 81-121. Berlin: Springer. 